\title{
Téoros
}

Revue de recherche en tourisme

\section{Canal de Lachine}

Expérience originale interuniversitaire et multidisciplinaire sur le développement du potentiel touristique du Canal de Lachine, berceau de l'industrialisation du Canada au $19^{\mathrm{e}}$ siècle

\section{François Bédard}

Volume 15, numéro 2, été 1996

Patrimoine industriel

URI : https://id.erudit.org/iderudit/1075028ar

DOI : https://doi.org/10.7202/1075028ar

Aller au sommaire du numéro

Éditeur(s)

Université du Québec à Montréal

ISSN

0712-8657 (imprimé)

1923-2705 (numérique)

Découvrir la revue

Citer cet article

Bédard, F. (1996). Canal de Lachine : expérience originale interuniversitaire et multidisciplinaire sur le développement du potentiel touristique du Canal de Lachine, berceau de l'industrialisation du Canada au $19^{\mathrm{e}}$ siècle. Téoros, 15(2), 46-48. https://doi.org/10.7202/1075028ar d'utilisation que vous pouvez consulter en ligne. 


\section{Canal de lachine}

\section{EXPÉRIENCE ORIGINALE INTERUNIVERSITAIRE ET MULTIDISCIPLINAIRE SUR LE DÉVELOPPEMENT DU POTENTIEL TOURISTIQUE DU CANAL DE LACHINE, BERCEAU DE L'INDUSTRIALISATION DU CANADA AU 19E SIĖCLE}

\author{
François Bédard \\ professeur-chercheur \\ Département d'études urbaines et touristiques de I'UQAM
}

Le présent article ténoigne d'une expérience pédagogique interuniversitaire et multidisciplinaire originale qui s"est deroulée entre 1993 et 1995 et dont l'objet portait sur le développement du potentiel touristique de la zone du Canal de Lachine laquelle fut le berceau de l'industrialisation du Canada au 19 e siècle.

L'équipe ayant participé à l'expérience comptait une quinzaine d'etudiants de l'Université McGill et de l'Université du Québec à Montreal inscrits dans quatre programmes différents: gestion, tourisme, urbanisme et anénagement urbain. La supervision du projel etait assuré par cinq professeurs provenant des deux universies.

\section{L'EXPERIENCE PEDAGOGIQUE}

L'expérience, laquelle a requis un an de planification et de préparation de la part des professeurs participant, a pris naissance à l'été 1993 à l'initialive de Lou D'Amore, président du connité organisatteur du deuxiême congrès mondial de l'Institut international pour la paix par le tourisme (IIPT) tenu à Montréal en septembre 1994. Sous le theme "Construire un monde durable par le tourisme ", le congrès regroupait des experts de plusieurs pays qui ont présenté des études de cas illustrant chacune un exemple réussi de développement durable en tourisme.

Désireux d'assurer des retombées concrètes du congrès dans la région mon-

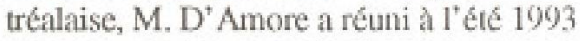
quelques professeurs d'université et leur a soumis l'idée que des étudirnts réalisent un projet de developpement durable en $s^{*}$ inspirant des exemples à succès présentés lors du congrès. Pour fâvoriser la col- lecte de donnés pertinentes, il a êté entendu que les étudiants joueraient un rôle actif at litre de rapporteurs d'ateliers lors du congrès, Lidlée a été retenue par les professeurs et un groune de travail a élé mis sur pied pour en assurer la réalisation.

La première tâche du groupe de travail a consisté à identifier les cours dams lespuels cente initiative serait insérée de manière à ce que le travail des éludiants soit crédité. Quelques reunions de planification ont permis de dégager un consensus pour retenir la zone du Canal de Lachine comme sujet principal de recherche. Après avoir passé en revue les cinq principaux poiles touristiques de Montreal (Biôdone-Jardin botanique-Pare Olympique : Parc des îles ; Vieux-Montrúal et Vieux-Port : Centre-ville ; Oratoire StJoseph el Mont Royal), il est apparu que le nouveau pôle des Rapides, incluạn le Canal de Lachine et son riche patrimoine industriel, était celui qui présentait le plus de potentiel de développement alu cours des prochaines années, et également celui qui revêtait le plus d' intérêt conme sujet d'ctude multidisciplinaire.

Pour des raisons pédagogiques et de fonctionnement, il a été décidé que le groupe d'une quinzaine d'étudiants serait divisé en quatre équipes ayant chacune la responsabilité d'un sujet spécifique d'étude relié au sujet principal. Les sujets d'études spécifiques retenus ont été : Le Marché Atwater et ses abords; la piste polyvalente du Canal de Lachine; le patrimoine industriel de la zone du Canal de Lachine; et le train touristique du Canal de Lachine.

Au début de la session de l'automne 1994, les êtudiants se sont vus remettre un document decrivant le contexte et les règles générales du projet incluant un court texte sur la problématique générale de chacun des quatre sujets d'études proposés. Les étudiants se sont regroupes en quatre équipes de travail. Chaque équipe jove le rôle d'une firme de consultants ayant reçu un mandat d'un client à qui le rapport linal de leur travail est destine. Ainsi l'équipe ayant réalisé l'étude sur le Marché Atwater a remis son rapport a la Corporation de Gestion des Marchés Publics de Montréal et au Regroupement pour la relance économique du Sud-Ouest (RESSO); celle de la piste polyvalente du Canal de Lachine a remis son travail à Pares Canada ; celle du patrimoine industriel de la zone du canal de Lachine a remis son travail à l'Office des congrès et du tourisme du Grand Montréal ; et finalement celle du train touristique du Canal de Lachine a remis son travail à RIFART, une firme privée vouée a la revalorisation des infrastructures ferroviaires pour des fins 
récréotouristiques. Une telle approche pédagogique s"est avérée avantageuse à plus d'un titre. D'une part, les étudiants y ont trouvé une grande motivation en rêalisant un apprentissage académique en étroite collaboration avec des intervenants du milieu. D'autre part, ces derniers ont reçu gracieusement un travail de qualité sur des problématiques les touchant directement.

Les résultats des études ont d'abord fait l'objet d'une présentation par' chacune des équipes lors d'une séance à laquelle participaient les representants des intervenants du milieu, les professeurs et les autres étudiants ayant participé à l'expérience. Finalement les études ont été officiellement rendues publiques lors du Sommet socioéconomique du Sud-Ouest de l'île de Montréal tenu à l'automne 1995. A cette occasion, les études, dont la valeur commerciale est estimée à quelque $100000 \$$, ont été remises aux représentants du nouveau pôle touristique des Rapides à titre de contribution des universités McGill et UQAM au développement touristique de la région Sud-Ouest de l'île de Montréal.

\section{SYNTHÈSE DE CHACUNE DES ÉTUDES}

Dans les lignes qui suivent, nous présentons une synthèse des quatre études réalisées.

\section{Le marché Atwater et ses abords}

La première étude porte sur le marché Atwater et ses abords.

Construit au cours des années 30, le marché Atwater constitue une véritable institution patrimoniale en bordure du Canal de Lachine. Jadis le lieu communautaire par excellence du quartier, son rayonnement comme marché public s'étend aujourd'hui au-delà de son voisinage immédiat. Il constitue I'un des fleurons du réseau des marchés publics montréalais. Sa localisation au coeur des premiers quartiers industriels du pays lui donne un caractère touristique enviable qui mérite d'être développé davantage. Ce n'est donc pas étonnant que le marché Atwatter ait été intégré au projet d'étude sur le potentiel touristique du Canal de Lachine.
L'étude propose des altematives quant à la mise en valeur des potentiels touristiques et communautaires du marchế Atwater tout en minimisant les contraintes operationnelles des activités commerciales actuelles. On y démontre que l'expansion des activités du marché Atwater par le biais d'un tourisme durable peut s'avérer un outil prometteur susceptible de contribuer à la relance de l'économie du SudOuest.

L'étude présente une description des cáractéristiques opérationnelles ainsi que de I'environnement physique du marché. On y présente également un inventaire des lieux d'intêrêt touristique dans le quartien et tinalement le protil socio-économique du quartier avoisinant. Puis on procedde à une analyse du potentiel touristique du secteur.

Le processus de consolidation du marché et de ses abords en un pöle touristique et communautaire se heurte à plusieurs contraintes d'ordre commercial, physique, touristique et social. Pour surmonter ces difficultés, et en vue d'élaborer une stratégie globale de développement touristique durable, un ensemble de solutions s'avere necessaire. Cette approche s'articule a travers les objectifs suivants : optimiser la fonctionnalité physique et commerciale du marché ; améliorer l'accès au site ; favoriser une exploitation optimale et durable des abords du marché ; développer des activités communautaires et touristiques au marché ; promouvoir la mise en place d'infrastructures complémentaires aux activités du marché.

Les stratégies proposées visent à promouvoir les activités commerciales du marché Atwater en les rendant plus accessibles et attrayantes pour la population du quartier tout en développant une activité touristique sur le site et ses abords qui sera profitable socialement et économiquement à la communauté locale.

En conclusion, l'étude souligne l'importance que le développement touristique se fasse de manière à maximiser les liens avec la population locale ainsi que les retombées sur celle-ci. Le marché Atwater pourrait être considéré comme une porte d'entrúe principale du tourisme dans le secteur. Le tourisme aurait un ellet positif sur la mise en valleur des richesses pitrimoniales et historiques du secteur et encouragerait la réaffirmation d'un sentiment positif d'appartenance pour la communauté, ce qui ne manquerait pas de stimuler la vie quotidienne de ces quartiers.

\section{La piste polyvalente du Canal de Lachine}

La deuxième étude porte sur la piste polyvalente du Canal de Lachine.

Le canal de Lachine est un lieu fort populaire. Quelque 500000 personnes le fréquentent annuellement, la grande majorité $(92 \%)$ pour la pratique du vélo. Les autres usages sont la promenade à pied $(6 \%)$ ou en patins à roues alignées $(2 \%)$. Les usagers de la piste polyvalente proviennent des quartiers avoisinants dans une proportion de $62 \%$.

Le premier plan directeur des abords du canal de Lachine a été élaboré par Parcs Canada en 1978. Le concept retenu visait la mise en valeur du site à partir d'un corridor đ'aclivilés terrestres et aquatiques reliant deux pôles historiques: le VieuxPort et le Vieux-Lachine. Bien que plusieurs aménagements aient étế ráalisés et que le pare du canal de Lachine soit en constante évolution, les objectifs et les projets définis à l'origine n'ont pu tous êre accomplis à ce jour. La composante principale du parc demeure la piste cyclable et les potentiels de mise en valeur et d'interprétation restent en grande partie sous-exploités.

Les auteurs de l'étude identifient deux problématiques posant un frein au développement du potentiel touristique de la piste polyvalente du canal de Lachine. Il y a d'abord la surutilisation de la piste cyclable, ce qui occasionne souvent des conflits entre les différents utilisateurs ainsi que certains problèmes de sécurité. En effet, il n'y a présentement que très peu d'intérêt pour les usagers de la piste polyvalente de faire des anrêts le long du parcours ou d'effectuer des sorties pour explorer les attraits des alentours. Le trafic reste donc concentré en majeure partie sur une bande relativement étroite le long du canal et la piste est utilisée comme un lieu de passage que l'on tente de traverser le plus rapidement possible. 
Le deuxième frein au développement touristique provient du manque d'attraits des lieux dans leur état actuel. En effet, le potentiel patrimonial des abords du canal ainsi que celui des quartiers avoisinants du canal $n^{\text {* }}$ ont pas encore fait l'objet d' une mise en valeur. Les écluses n'ont pas été restaurées, et les éléments d'interprétation des lieux historiques sont quasi inexistants. La signalisation est insuffisante pour des visiteurs non familiers avec les lieux.

Les auteurs de l'étude concluent en proposant une priorisation de certains utilisateurs en fonction des differrents grands tronçons le long du canal s"étendant sur une distance d"environ douze kilomètres. Ils identifient des secteurs plus propices à attirer des touristes, d'autres plus propices a la pratique d'activités récréatives, et enfin d'autres plus propices à la pratique d'activités sportives.

\section{Le train touristique du Canal de Lachine}

La troisième étude porte sur un projet de train touristique reliant le Vieux-Port et le Canal de Lachine.

L'accessibilité au canal Lachine n'est pas une chose évidente pour les touristes qui séjournent à Montréal sauf pour ceux qui empruntent la piste cyclable à partir du Vieux-Port de Montréal. L'idée d"utiliser pour des fins touristiques le lien ferroviaire déjà existant entre le Vieux-Port et le Canal de Lachine vise à inciter le plus de visiteurs possibles, parmi les quatre millions qui frequentent le Vieux-Port au cours de la saison estivale, à découvrir la zone du Canal Lachine.

Le concept du train proposé représente bien davantage qu'un simple moyen de transport collectif reliant deux points entre eux. Il constitue une nouvelle attraction touristique s'ajoutant à celles dẹjà présentes dans le Vieux-Port. La locomolive et les wagons ont un caractère patrimonial. Les passagers sont accueillis a bord du train par un persomel d'accueil en costume d'époque. Tout le long du parcours, l'histoire des lieux est racontée de façon animee. Pendant l'heure que dure le trajet aller-retour du Vieux-Port au Square SaintPatrick (premier tronçon alyaunt lkit l'ohjet de la présente étude de pré-faisabilité), les passagers vivent une aventure à la lois divertissante et éducative sur le patrimoine montréalais.

L'étude de pré-faúsabilité comporte une évaluation de l'environnement sociopolitico-économique dans lequel le projet se situe. Des consultations préliminaires aupres des intervenants des milieux concernés ont démontué qu"il y avaít un intérêt pour un tel projet dans la mesure où la faisabilité est dêmontrée.

Se référant à lã grande popularité que plusieurs trains d'époque connaissent filleurs en Amérique du Nord, on peut s'attendre à ce que le train proposé fasse la joie de nombreux jeunes et de moins jeunes qui fréquentent le Vieux-Port. On estime à quelque 90000 passagers annuellement le potentiel d'achalandage pour un train touristique reliant le VieuxPort et le Canal de Lachine.

Un certain nombre d'éléments freins à la réalisation du projet sont toutefois identifiés, notamment les conditions d'utilisation des lignes ferroviaires existantes, les coûts d'acquisition ou de réfection du matériel roulant ă caractère patrimonial, et la formation d'un groupe de promoteurs ayant la capacité de rónliser le montage financier du projes.

\section{Le patrimoine industriel de la zone du Canal de Lachine}

La quatrième étude porte sur le patrinoine industriel de la zone du Canal de Lachine.

L'étude présente un cadre de référence pour la planification et le développenent du pitrimoine industriel de la zone du Ganal de Lachine. Elle propose de développer celte zone en écomuséc. Plutôt que de placer l'accent sur des sites patuimoniaux spéciliques, l'écomusée fonctionnerail comme grand musếe naturel voué à da connaiseance ot à l'interpréciation de l'ensemble de ce territoire. Plus spécifiquement, l'écomusée illustrerait comment la révolution industrielle đu $19 \mathrm{e}$ siecle et du début du $20 \mathrm{e}$ siecle a façonné les aspects physiques de ce territoire, et comment elle a influence les comportements sociaux et culturels des gens qui $y$ vivaient.
L'étude élabore un processus de développement nécessaire pour supporter un programme d'intervention durable, présente des lignes directrices visant l'implication des intervenants du milieu et propose la mise sur pied d'une structure de coordination qui superviserait le développement de l'ensemble de la zone du canal.

Finalement l'étude évoque le rôle que remplirait cet écomusée parmi les autres maillons du mini-réseau rếgional des pôles à caractère historique et patrimonial dans la région de Montréal (ex. VieuxMontréal, Vieux-Port, Vieux-Lachine).

\section{BIBLIOGRAPHIE}

Héroux, Jocelyne ; Morin, Stéphane ; Trempe, Delphine : Plan de mise en valeur de la piste polyvalenie du canal de Lachine, MeGill, 1995.

Huard, Helene al al, : The Tourist Train : A preFeasibility Study, Final Report Submitted to : RIFART, Fall 1994.

Le groupe CDFK, Consultants en urbanisme, Le manche Afwater et ses abords: Towrisne durable et stwitegies de développement, rapport final soumis a Corporation de Gestion des Marchés Publics de Montréal et RÉSO, 1995.

UtbEco Planning Consultants $s_{\text {q }}$ Utten Revitalization Though Tourism : Indastrial Herilage and the Lachine Canal Corridor, Final Report Submitted to : I'Ofice des Congres at du Tourisme du Grand Montral (OCTGM), 1995 